\title{
Formation of protein kinase C $\varepsilon$-Lck signaling modules confers cardioprotection
}

\author{
Peipei Ping, ${ }^{1,2}$ Changxu Song, ${ }^{1}$ Jun Zhang, ${ }^{1}$ Yiru Guo, ${ }^{2}$ Xinan Cao, ${ }^{1,2}$ Richard C.X. Li, ${ }^{1,2}$ \\ Wenjian Wu, ${ }^{2}$ Thomas M. Vondriska, ${ }^{1,2}$ Jason M. Pass, ${ }^{1,2}$ Xian-Liang Tang, ${ }^{2}$ \\ William M. Pierce, ${ }^{3}$ and Roberto Bolli ${ }^{1,2}$ \\ ${ }^{1}$ Department of Physiology and Biophysics, \\ ${ }^{2}$ Department of Medicine, Division of Cardiology, and \\ ${ }^{3}$ Department of Pharmacology and Toxicology, University of Louisville, Louisville, Kentucky, USA \\ Address for correspondence: Peipei Ping, 570 South Preston Street, Baxter Building, \\ Suite 122, Cardiology Research, Louisville, Kentucky 40202-1783, USA. \\ Phone: (502) 852-8431; Fax: (502) 852-8421; E-mail: peipeiping@hotmail.com.
}

Received for publication May 4, 2001, and accepted in revised form January 14, 2002.

\begin{abstract}
The $\varepsilon$ isoform of protein kinase $\mathrm{C}(\mathrm{PKC} \varepsilon)$ is a member of the $\mathrm{PKC}$ family of serine/threonine kinases and plays a critical role in protection against ischemic injury in multiple organs. Functional proteomic analyses of $\mathrm{PKC} \varepsilon$ signaling show that this isozyme forms multiprotein complexes in the heart; however, the precise signaling mechanisms whereby PKCE orchestrates cardioprotection are poorly understood. Here we report that Lck, a member of the Src family of tyrosine kinases, forms a functional signaling module with PKCE. In cardiac cells, PKCE interacts with, phosphorylates, and activates Lck. In vivo studies showed that cardioprotection elicited either by cardiac-specific transgenic activation of PKCE or by ischemic preconditioning enhances the formation of PKC $\varepsilon$-Lck modules. Disruption of these modules, via ablation of the Lck gene, abrogated the infarct-sparing effects of these two forms of cardioprotection, indicating that the formation of PKCE-Lck signaling modules is required for the manifestation of a cardioprotective phenotype. These findings demonstrate, for the first time to our knowledge, that the assembly of a module (PKCe-Lck) is an obligatory step in the signal transduction that results in a specific phenotype. Thus, PKCE-Lck modules may serve as novel therapeutic targets for the prevention of ischemic injury.
\end{abstract}

J. Clin. Invest. 109:499-507 (2002). DOI:10.1172/JCI200213200.

\section{Introduction}

Ischemic injury can result in cell death and irreversible loss of function in a variety of biological systems (1-3). An understanding of the intracellular signaling mechanisms by which cells protect themselves against ischemia-induced damage bears great clinical significance with respect to the treatment and prevention of tissue injury (1-3). The $\varepsilon$ isoform of protein kinase $C$ (PKCE) is a member of the PKC family of serine/threonine kinases and has been implicated in the protection against injury in multiple organ systems (4-13). In the heart, activation of PKC $\varepsilon$ has been shown to mediate the development of preconditioning $(4,5$, 11-13), a powerful cardioprotective adaptation that can be produced either by brief episodes of ischemia followed by reperfusion, or by the administration of pharmacological agents that can mimic the effects of ischemic preconditioning $(1,14-18)$. However, the manner in which this individual kinase (PKCE) interacts with other proteins within the signaling system to conduct signal transduction during cardioprotection remains unknown.

The colocalization of multiple proteins to transmit subcellular signals is an emerging theme underlying recent studies in signal transduction (19-25). For instance, a number of investigations have characterized the regulation of mitogen-activated protein kinase (MAPK) cascades by scaffolding proteins $(22,24,25)$. Another paradigm that embodies a theme of compartmentalization is the assembly of multiprotein signaling complexes $(19,20)$. We recently reported that, in the murine myocardium, PKCE forms complexes with at least 36 proteins that can be categorized as structural proteins, signaling kinases, and stress-responsive proteins (19). The composition of this complex is dynamically altered during cardioprotection (19), indicating that the regulation of complex components may serve as a means for cardioprotective signaling. One of the signaling kinases identified in this study was Lck, a member of the Src family of nonreceptor tyrosine kinases that has been characterized as a central signaling molecule in numerous biological systems (26-28). The recruitment of Lck to the PKC $\varepsilon$ signaling complex was associated with posttranslational modification of Lck during cardioprotection (19), suggesting that this tyrosine kinase may function in concert with PKCe to confer a specific phenotype. Given the biological ubiquity of PKC $\varepsilon$ and Lck, it is of particular importance to understand how these molecules might interact to perform multiple signaling tasks. 
Although the biological roles of both PKCE and Lck have been extensively studied in a variety of systems $(4-13,26-33)$, it is unknown whether these two kinases interact and, if so, in which specific manner they interact, and whether their coordinated interactions are required to govern signal transduction during the genesis of a biological phenotype (such as protection against ischemic injury). In the present investigation, we found that PKCE interacted with, phosphorylated, and activated Lck; that is, PKC $\varepsilon$ and Lck functioned as a signaling module. Furthermore, using a well-established in vivo model of regional myocardial ischemia, we found that both chronic activation of PKCE (PKCE transgenesis) and acute activation of PKCE (ischemic preconditioning) promoted the formation of PKC $\varepsilon$-Lck modules and evoked cardioprotection. Disruption of these modules, via deletion of the Lck gene, abrogated the infarct-sparing effects of both of these forms of cardioprotection, demonstrating that these modules play a causative role in the reduction of myocardial infarct size. Based on these lines of evidence, we propose that the formation of signaling modules may be a common mechanism employed by different signaling systems in order to facilitate the development of their respective biological phenotypes. Furthermore, our data indicate that pharmacological interventions targeted at the formation of these modules may be utilized for the prevention of ischemic injury.

\section{Methods}

Transgenic mice. PKCE transgenic mice were generated (ICR background) using a cDNA of active PKCE driven by the $\alpha$-myosin heavy chain promoter to achieve cardiac-specific expression $(10,34)$. The PKC $\varepsilon$ transgenic line used in these studies expresses low levels of PKCE and is free of cardiac hypertrophy (10). Breeding pairs of $\mathrm{Lck}^{-/-}$mice (C57BL/6J) were obtained from The Jackson Laboratory (Bar Harbor, Maine, USA). To obtain PKCE-Lck $k^{-/-}$mice, PKC $\varepsilon$ mice were mated with $L c k^{-/-}$ mice, and the $\mathrm{F}_{2}$ through $\mathrm{F}_{4}$ chimeric offspring were genotyped, via PCR and Southern blot analysis, to document PKC $\varepsilon$ transgene transmission and deletion of the Lck gene. $\mathrm{F}_{4}$ offspring were used in this study. Although the genetic background of these PKC $-\mathrm{Lck}^{-/-}$mice (approximately $80 \% \mathrm{C} 57 \mathrm{BL} / 6 \mathrm{~J}$ ) differs from that of either parent strain (ICR and C57BL/6J), in previous studies we have found that the response of ICR and C57BL/6J mice both to a 30-minute coronary occlusion and to ischemic preconditioning is indistinguishable. Consequently, it is highly unlikely that the differences in infarct size between PKCE- $L \mathrm{Ck}^{-/}$mice and PKC $\varepsilon$ mice are accounted for solely by differences in the genetic background. The PKCE, $L c k^{-/}$, and PKCE-Lck ${ }^{-/}$mice and their wild-type controls were studied at age 9-12 weeks. Mouse model of myocardial infarction. The murine model of ischemic preconditioning and myocardial infarction has been described in detail (35). Briefly, myocardial infarction was produced by a 30-minute coronary occlusion followed by a 4-hour reperfusion. To induce ischemic preconditioning, mice underwent a sequence of six 4-minute coronary occlusions separated by $4 \mathrm{~min}$ utes of reperfusion, 24 hours prior to the 30 -minute occlusion. Fundamental physiological variables that impact on infarct size, including rectal temperature, arterial blood pressure, heart rate, and arterial blood gases, were carefully monitored and maintained within normal limits (35). At the conclusion of the study, the occluded/reperfused vascular bed and the infarct were identified by postmortem perfusion. Infarct size was calculated by computerized videoplanimetry (35).

Recombinant PKCE and Lck proteins. Mouse cDNAs encoding wild-type PKCE, the catalytic domain (CHA) of PKCE, the regulatory domain (RHA) of PKCE, or wildtype Lck were cloned into the PAcGHLT vector, expressed in the baculovirus system, and purified to generate glutathione S-transferase (GST) fusion proteins (BD PharMingen, San Diego, California, USA). GST fusion proteins with various Lck domains (GST-Unique, amino acids [a.a.] 1-67; GST-SH2, a.a. 67-116; GST-SH3, a.a. 116-238) were obtained from BD PharMingen. Cold or $\left[{ }^{35} \mathrm{~S}\right]$ methionine-labeled recombinant proteins of PKC $\varepsilon$ wild-type, PKC - -RHA, PKC $\varepsilon$-CHA, and Lck wildtype were also made through in vitro transcription and translation using the TNT Quick-Coupled rabbit reticulocyte lysate system (Promega Corp., Madison, Wisconsin, USA). The GST-PKCE, GST-Lck, and the in vitro translated PKC $\varepsilon$ and Lck were all verified to retain their kinase activity (data not shown).

Assessing protein-protein interactions via coimmunoprecipitation and GST affinity pull-down assays. Immunoprecipitation assays were performed using PKC $\mathrm{mAb}$ 's (BD PharMingen). Nonspecific bead-associated proteins were initially precleared from the tissue homogenates by the addition of protein-G beads. After the preclearing step, the sample was split into equal portions. For immunoprecipitation of the PKCe complex, PKCe mAb's were added to the precleared homogenates. Nonspecific interactions were identified by substituting IgG for the PKCE antibodies.

GST recombinant protein-based affinity pull-down assays were performed as previously reported (19). Briefly, GST recombinant proteins (e.g., GST-PKCE) were immobilized on GST beads, mixed with either tested recombinant proteins or cardiac tissue homogenates, and incubated in binding buffer $(0.5 \%$ Triton $X-100,20$ mM Tris-HCl, pH 7.4, 1 mM EDTA, 1 mM EGTA, 0.2 $\mathrm{mM}$ sodium orthovanadate, $1 \times$ proteinase cocktail [Roche Molecular Biochemicals, Indianapolis, Indiana, USA]) with various concentrations of $\mathrm{NaCl}$. The reactions were terminated by a 5 -minute centrifugation at $500 \mathrm{~g}$. The GST-protein pellets were washed, resolved by SDS-PAGE, and analyzed via immunoblotting with antibodies against corresponding proteins. To determine nonspecific binding, parallel reactions were conducted using equal molar amounts of GST-null proteins in lieu of the tested GST fusion proteins.

The binding affinities between PKCE and Lck were assessed via an ELISA-based binding assay. Polystyrene 
96-well plates (Corning-Costar Corp., Cambridge, Massachusetts, USA) were coated with purified GST-Lck (0, 50, 200, 400, $800 \mathrm{ng})$, washed with TBST buffer (20 mM Tris-HCl, pH 7.4, $150 \mathrm{mM} \mathrm{NaCl}, 0.05 \%$ Tween-20), and blocked with $1 \%$ BSA. Various amounts of hemagglutinin-tagged (HA-tagged) and in vitro translated HA-PKCE-CHA or HA-PKCE-RHA $(10,55,150,300 \mathrm{ng})$ were blotted onto the plates. After incubation, the plates were washed with TBST and incubated with HA antibodies (Berkeley Antibody Co., Richmond, California, USA). The reactions were washed with TBST, developed with 3,3',5,5'-tetramethylbenzidine, and treated with $2 \mathrm{M} \mathrm{H}_{2} \mathrm{SO}_{4}$. Lck and PKCE binding was quantified using OD reading at $450 \mathrm{~nm}$ (Microplate Reader; Bio-Rad, Hercules, California, USA).

In vitro phosphorylation of Lck by PKCE. Kinase active $\mathrm{PKC} \varepsilon$ recombinant proteins were incubated with wildtype or various GST-Lck mutants in the PKC phosphorylation cocktail $(50 \mathrm{mM}$ Tris- $\mathrm{HCl}[\mathrm{pH} 7.5], 0.1$ $\mathrm{mM}$ ATP, $2.3 \mathrm{mM}$ HEPES, $28.8 \mu \mathrm{g} / \mathrm{ml}$ L- $\alpha$-phosphatidyl-L-serine, $3 \mu \mathrm{g} / \mathrm{ml}$ phorbol PMA, $7.5 \mathrm{mM}$ DTT, $3.0 \mathrm{mM}$ calcium acetate, $5.5 \mathrm{mM} \mathrm{MgCl}_{2}$, and $0.5 \mu \mathrm{Ci}$ $\left[\gamma^{32} \mathrm{P}\right]$ ATP) (12). Phosphorylation of Lck was analyzed via SDS-PAGE and autoradiography.
$P K C \varepsilon$ and Lck activity assays. The phosphorylation activity of PKC $\varepsilon$ was determined as previously described (12). Briefly, tissue protein samples were immunoprecipitated overnight with PKCE antibodies (BD PharMingen). The immunoprecipitates were incubated with the PKCE-selective substrate (ERMRPRKRQGSVRRRV) in the PKC phosphorylation cocktail (12).

The phosphorylation activity of Lck was determined as previously described (32). Briefly, protein samples were immunoprecipitated with Lck antibodies (Santa Cruz Biotechnology Inc., Santa Cruz, California, USA), and the immunoprecipitates were subjected to an Src tyrosine kinase phosphorylation assay using either the peptide (KVEKIGEGTYGVVYK) or enolase (SigmaAldrich, St. Louis, Missouri, USA) as substrates in an Src phosphorylation cocktail (32). The phosphorylation activity of PKC $\varepsilon$-associated Lck was determined by subjecting the PKC $\varepsilon$-immunoprecipitated complex to the Lck kinase phosphorylation assay.

\section{Results}

PKCE and Lck form functional signaling modules in the murine myocardium. We first sought to determine whether Lck is able to physically interact with PKCe. Consistent with our previous report, we found that a
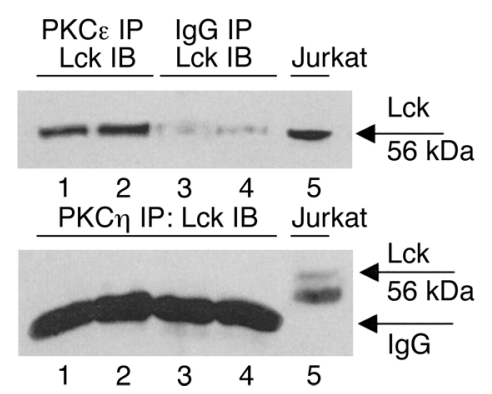

d

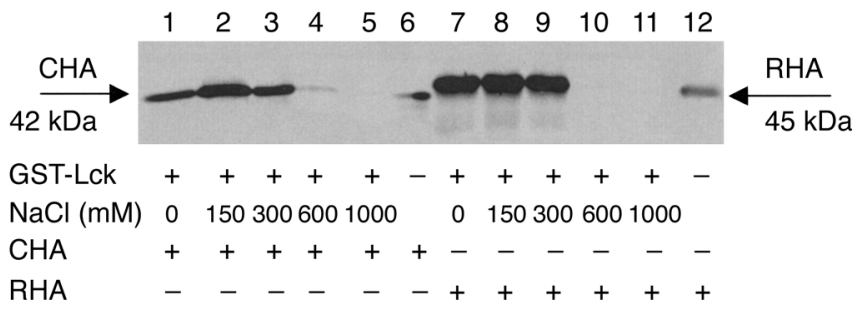

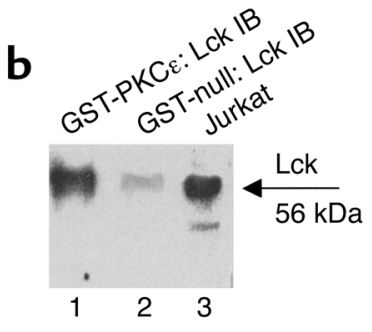

C

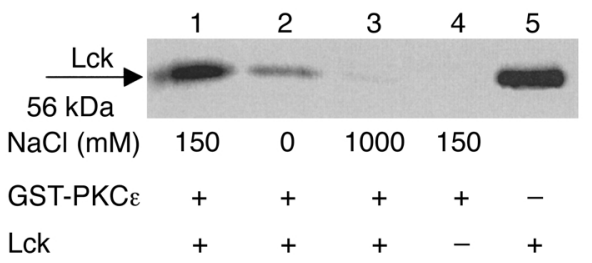

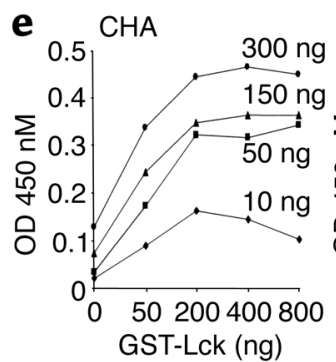

RHA

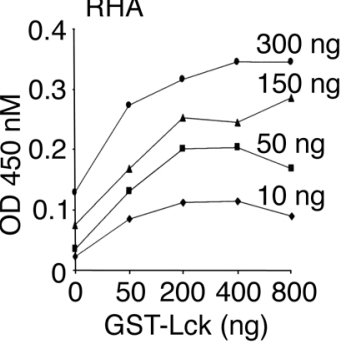

Figure 1

PKCE exhibits direct physical interactions with Lck in vivo and in vitro. (a) Lck co-resided with PKCE (upper panel) but not with PKC $\eta$ (lower panel). Upper panel: $200 \mu \mathrm{g}$ proteins were immunoprecipitated with either PKCE mAb's (lanes 1 and 2) or IgG (lanes 3 and 4 ) and immunoblotted with Lck antibodies. Lane 5 served as positive control. Lower panel: $200 \mu \mathrm{g}$ proteins were immunoprecipitated with the PKC $\eta$ polyclonal antibodies and immunoblotted with Lck antibodies (lanes 1-4). Lane 5 served as positive control. (b) Mouse myocardial Lck interacted with GST-PKCE. Lck expression was detected in cardiac tissue by GST-PKCE pull-down (lane 1). GST-null vector was negative control (lane 2). Lane 3 served as positive control. (c) PKCE exhibited physical interactions with Lck in vitro. Recombinant GST-PKCE proteins were incubated with in vitro translated and [ $\left.{ }^{35} \mathrm{~S}\right]$ methionine-labeled Lck. Lanes 1-3 contain GST-PKC $\varepsilon$ and Lck with various concentrations of $\mathrm{NaCl}$; lane 4 served as negative control without Lck; lane 5 served as the $\left[{ }^{35} \mathrm{~S}\right]$ methionine-labeled Lck (positive control). (d) Lck interacted with both the catalytic (CHA) and the regulatory (RHA) domains of PKCE. GST-Lck proteins were incubated with in vitro translated and [ $\left.{ }^{35} \mathrm{~S}\right]$ methionine-labeled CHA and RHA proteins. Lanes 1-5 depict GST-Lck pull-down of CHA with various concentrations of $\mathrm{NaCl}$; lane 6 served as positive control (CHA proteins); lanes 7-11 depict GST-Lck pull-down of RHA with various concentrations of $\mathrm{NaCl}$; lane 12 served as positive control (RHA proteins). (e) ELISA-based binding assays were performed to determine Lck interactions with CHA and RHA. GST-Lck was found to bind to CHA proteins (left) and RHA proteins (right) with similar affinity. 


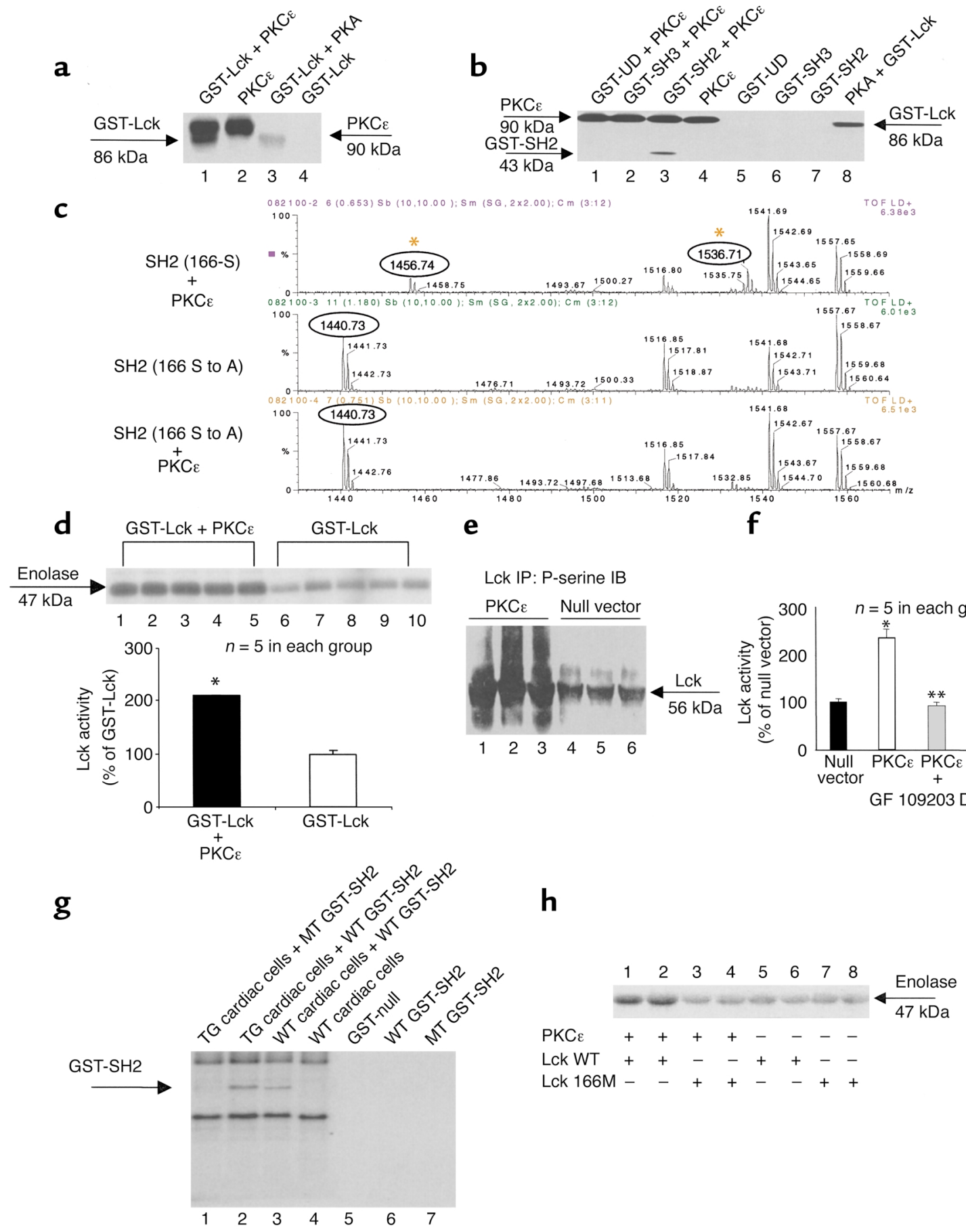

\section{Figure 2}

PKC $\varepsilon$ and Lck form functional signaling modules. (a) PKCE phosphorylated GST-Lck. Lane 1: GST-Lck (86 kDa) and autophosphorylated PKCE (90 kDa); lane 2: autophosphorylated PKCE; lane 3: GST-Lck phosphorylated by PKA; lane 4: GST-Lck alone (negative control). (b) PKC $\varepsilon$ phosphorylates the SH2 domain, but not the GST-UD and GST- SH3 domains of Lck (lanes 1and 3). Lane 8 shows phosphorylation of GST-Lck by PKA (positive control). (c) PKC $\varepsilon$ phosphorylates S166 of Lck in vitro. Examples of mass spectrums. Upper panel: PKCE phosphorylates the $\mathrm{SH} 2$ domain (the phosphorylated peak [ $\mathrm{m} / \mathrm{z}$ value] of 1536.71 and the unphosphorylated peak of 1456.74). Middle panel: The mutated SH2 domain (the unphosphorylated peak of 1440.73 [S-A mutation shifted the $\mathrm{m} / \mathrm{z}$ value]). Lower panel: PKC $\varepsilon$ does not phosphorylate the mutated SH2 (the unphosphorylated peak of 1440.73). (d) PKCE enhanced Lck activity in vitro. Lck activity was determined by phosphorylation of either enolase (upper panel) or Src substrates (lower panel). ${ }^{*} P<0.01$ vs. GST-Lck. (e) Activation of PKC $($ lanes $1-3$ ) enhanced serine phosphorylation of Lck. Negative control with null vector (lanes 4-6). (f) Activation of PKC $\varepsilon$ via PKC $\varepsilon$-adenoviruses enhanced Lck activity, which was blocked by GF109203, or by a PKC $\varepsilon$ dominant negative mutant (DN-PKCE) $(12)$. ${ }^{*} P<0.05$ vs. null vector, ${ }^{*} P<0.05$ vs. PCKE. (g) Myocardial PKCE phosphorylates the SH2 domain of Lck. Transgenic (TG) PKCE enhances phosphorylation of wildtype (WT) SH2 (lane 2 versus lane 3), but not that of the mutant (MT) SH2 (lane 1). (h) Mutation of SH2 domain reduces Lck activity. PKCE-dependent Lck activity was reduced with SH2 mutant (Lck $166 \mathrm{M}$, lanes 3 and 4) compared with that of the wild-type SH2 (Lck WT, lanes 1 and 2). Data are mean \pm SEM. 
Lck co-resided in the PKCE complex both in cardiac myocytes (as demonstrated by coimmunoprecipitation; Figure 1a) and in the myocardium (as illustrated by GST-PKC $\varepsilon$ pull-down assays; Figure $1 \mathrm{~b}$ ). In contrast, the expression of Lck was not detected in PKCn immunocomplexes (Figure 1a), indicating that the association of Lck and PKCE is isoform-specific. Furthermore, GST-PKCE exhibited physical interactions with recombinant Lck proteins in an $\mathrm{NaCl}$ concentration-dependent fashion in vitro (Figure 1c), indicating that the interactions are ionic in nature. ELISA-based binding assays showed that GST-Lck binds to both CHA proteins (left panel) and RHA proteins (right panel) with similar affinity (Figure 1e). Collectively, these data indicate that Lck is a binding partner of PKCE.

Next, we examined whether physical interactions between PKCE and Lck facilitate the transduction of a signal. To address this issue, we tested whether PKCE can transfer a signal to Lck by direct chemical modification, i.e., whether PKCE and Lck compose a functional signaling module. In the in vitro setting, PKCE was found to phosphorylate recombinant Lck proteins (Figure 2a). Using recombinant proteins carrying various mutations of Lck, phosphorylation by PKC $\varepsilon$ was found to be localized exclusively to the $\mathrm{SH} 2$ domain of Lck (Figure 2b). Matrix-assisted laser desorption ionization (MALDI) mass spectrometric analysis revealed that, among the four PKC recognition motifs harbored within the SH2 domain, the serine 166 site was phosphorylated in the presence of active PKC $\varepsilon$ in vitro (Figure $2 \mathrm{c}$ ). Point mutation of this site (S to $\mathrm{A}$ ) abolished the phosphorylation signal (Figure 2c). Importantly, PKC $\varepsilon$ phosphorylation of the recombinant Lck proteins was associated with a commensurate increase in the kinase activity of Lck as demonstrated both by GST-Lck-induced phosphorylation of enolase and by GST-Lck-induced phosphorylation of the Src-specific peptide KVEKIGEGTYGVVYK (Figure 2d). In both of these experiments, which utilized completely different substrates for Lck, GST-Lck exhibited significantly higher activity in the presence of PKC $\varepsilon$ when compared with that in the absence of PKCE (GST-Lck alone). To verify these findings in intact cells, cardiac myocytes were transfected with recombinant adenoviruses encoding active PKCE. Activation of PKC 8 in these cells resulted in increased serine phosphorylation of Lck (Figure 2e) and elevated Lck activity (Figure 2f). In addition, we performed phosphorylation assays using endogenous myocardial PKCE. Our results show that myocardial PKCE phosphorylates recombinant $\mathrm{SH} 2$ domains and that mutation of the S166 residue in the SH2 domain diminishes this phosphorylation event (Figure 2g) and attenuates
PKCE-dependent Lck phosphorylation activity (Figure 2h). Taken together, these data demonstrate that PKC $\varepsilon$ and Lck form a signaling module in vitro and in cardiac myocytes, and that the formation of this PKCE-Lck signaling module serves to facilitate signal transduction between PKCE and Lck.

Transgenic activation of PKCE protects against myocardial infarction. Brief episodes of ischemia/reperfusion render the heart remarkably resistant to a subsequent lethal ischemic insult, a phenomenon termed ischemic preconditioning $(1,14)$. The $\varepsilon$ isozyme of $\mathrm{PKC}$ has been implicated as an essential signaling element in the genesis of ischemic preconditioning $(4,5,11-13)$. To determine whether activation of PKCE in itself is sufficient to recapitulate the preconditioned phenotype, we generated a transgenic mouse line expressing cardiac-targeted active PKC $\varepsilon$ (these mice are free of cardiac hypertrophy) (10). In the myocardium of these PKC $\varepsilon$ mice, the level of PKC $\varepsilon$ activation (Figure 3a) and the extent of PKCE translocation (Figure 3b) fully mimicked those elicited in wild-type mice by ischemic preconditioning (six cycles of 4-minute coronary occlusion separated by 4 minutes of reperfusion) (35) (Figure 3, a and b). In accordance with our previous studies (35), the size of the infarct induced by a 30 -minute coronary artery occlusion was markedly reduced in ischemically preconditioned wild-type mice when compared with nonpreconditioned controls (Figure 4, a and b). In PKC $\varepsilon$ transgenic mice subjected to the same 30-minute coronary occlusion without ischemic preconditioning, myocardial infarct size was significantly less than in nontransgenic mice (Figure 4, a and c) and was similar to that noted in preconditioned wild-type mice (Figure 4, a and c). These data demonstrate that the PKCE mice are inherently resistant to ischemic injury, thereby displaying a cardioprotective phenotype similar to that a
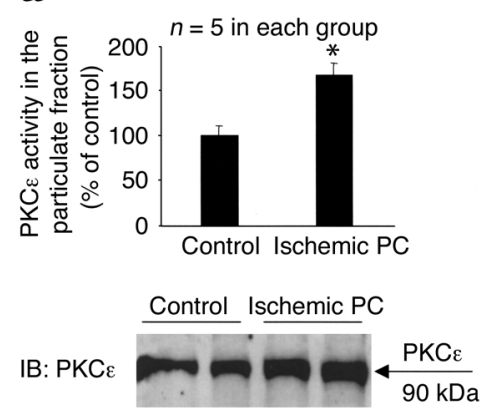

Figure 3

Ischemic preconditioning (PC) and PKCE transgenesis induce activation and translocation of PKCE. (a) Wild-type mice that underwent an ischemic PC protocol (six cycles of 4-minute coronary occlusion and 4-minute reperfusion) exhibited enhanced phosphorylation activity of PKCE (upper panel) concomitant with increased particulate expression of the PKC $\mathrm{P}$ protein (lower panel) when compared with wild-type mice that underwent a sham operation (control). Tissue samples were harvested 30 minutes after the last reperfusion or at corresponding times in controls. ${ }^{*} P<0.05$ vs. control. (b) Transgenic mice carrying a cardiac-targeted active PKC $\varepsilon$ cDNA displayed increased $\mathrm{PKC} \varepsilon$ protein and $\mathrm{PKC} \varepsilon$ activity in the particulate fraction when compared with wild-type mice. Data are mean \pm SEM. ${ }^{*} P<0.05$ vs. wild-type. 
a
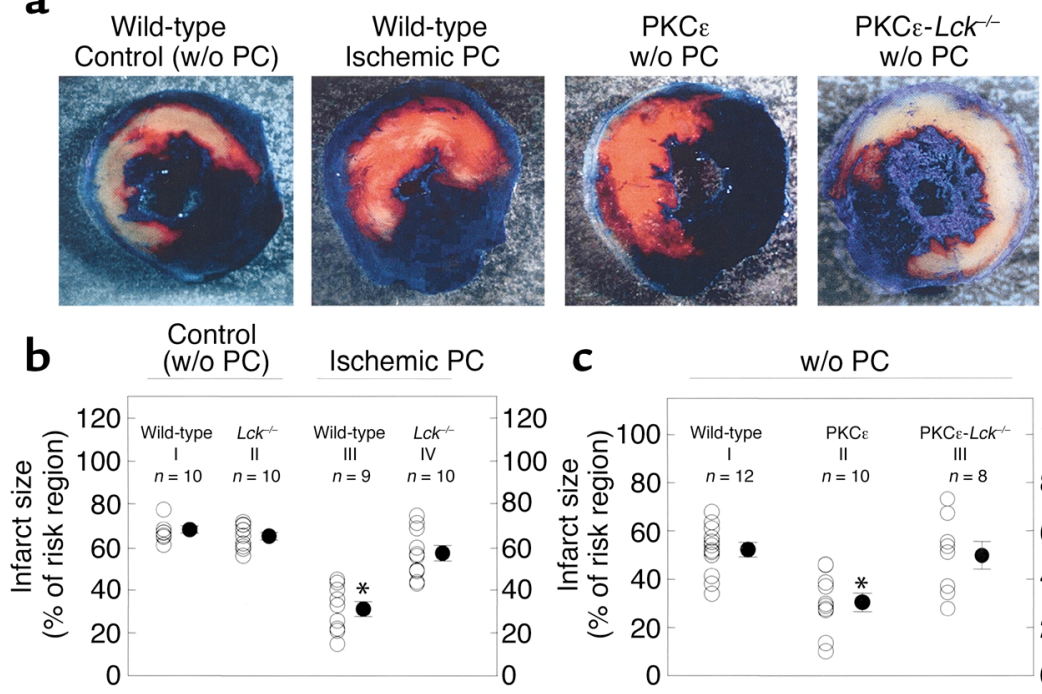

C

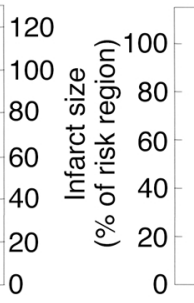

\section{Figure 4}

Ablation of the $L c k$ gene abrogates ischemic preconditioning (PC) and PKCE-induced cardioprotection. (a) Mice underwent a 30-minute coronary occlusion followed by a 4-hour reperfusion. After postmortem perfusion, the nonischemic portion of the left ventricle was stained dark blue and the viable tissue within the region at risk bright red, whereas the infarcted tissue was stained light yellow. Illustrated are representative examples of infarcts observed in wildtype mice subjected to sham surgery (controls), wild-type mice subjected to ischemic PC, PKCE transgenic mice, and double mutant mice expressing active PKC $\varepsilon$ with ablation of the $L c k$ gene. Myocardial infarction was markedly reduced in the wild-type preconditioned mouse and in the $\mathrm{PKC} \varepsilon$ mouse not subjected to ishcemic PC. However, in the PKC $\varepsilon-L c k^{-1-}$ mouse, the extent of infarction was similar to the control mouse. (b) Quantitative analysis of infarct size in wild-type and $L c k^{-/-}$mice. Infarct size was similar in wild-type and $L c k^{-/-}$non-preconditioned mice, indicating that basal LCK activity does not modulate the severity of ishcemia/reperfusion injury. When mice were subjected to ischemic PC, infarct size was reduced in wild-type but not in $\mathrm{Lck}^{-/}$ mice, indicating that Lck is an obligatory signaling component in the genesis of late ischemic PC. ${ }^{*} P<0.05$ vs. group I. (c) Quantitative analysis of infarct size in wild-type, PKC $\varepsilon$ transgenic, and PKCE-LCk ${ }^{-1}$ mice. Activation of PKCE reduced infarct size to $31.0 \% \pm 3.6 \%$ of the risk region. This protective effect of PKC $\varepsilon$ transgenesis was absent in PKC $\varepsilon-L c K^{-/-}$mice, indicating that PKCE-induced cardioprotection requires $L c k$. ${ }^{*} P<0.05$ vs. group I. Data are mean \pm SEM.

exhibited by wild-type mice preconditioned with ischemia. The magnitude of the protection afforded by selective activation of PKC $\varepsilon$ appears to be equivalent to that afforded by ischemic preconditioning.

PKCE-Lck signaling modules play an obligatory role in cardioprotection. Having characterized the functional significance of the PKCE-Lck signaling modules (enhanced signal transduction involving these two molecules), we then proceeded to assess the physiological impact that these modules exert in vivo. To determine the role of Lck and PKCE-Lck signaling modules in the genesis of cardioprotection, we examined the activity of Lck, the formation of PKCE-Lck signaling modules, and the extent of cardiac cell death (infarction) in each of the following groups of mice: wild-type mice, ischemically preconditioned mice, PKC $\varepsilon$ transgenic mice, mice with targeted ablation of the Lck gene $\left(L c k^{-/}\right)$, and double mutant mice exhibiting cardiacspecific expression of active PKCE in addition to deletion of the $L c k$ gene (PKC $\varepsilon-L c k^{-/}$).

In both ischemically preconditioned wild-type mice and PKC $\varepsilon$ cardioprotected mice, we observed enhanced w/o PC

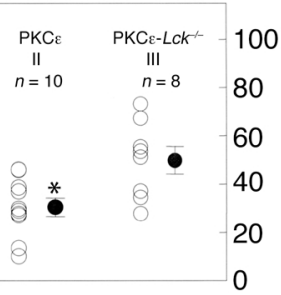

localization of Lck to the particulate $\mathrm{PKC \varepsilon}$ complex that was accompanied by increased Lck activity (Figures 5, a and b). Thus, augmentation of PKCe-Lck signaling module formation was congruous with the cardioprotective effects conferred by both ischemic preconditioning and $\mathrm{PKC} \varepsilon$ activation (Figure 4, a-c). Next, we determined whether these modules are required for cardioprotection. Accordingly, double mutant mice exhibiting cardiac specific transgenic activation of PKCE along with deletion of the Lck gene were generated (Figure 5c). We found that, in contrast to wildtype mice, ischemic preconditioning produced no infarct-sparing effects in $L c k^{-/-}$mice, and that the reduction in infarct size observed in PKCE mice was completely lost in PKCE-Lck $k^{-/-}$mice (Figure 4, a-c), indicating that ablation of the Lck gene abrogated both forms of cardioprotection. To our knowledge, these results provide the first indication that Lck-dependent signaling plays a necessary role in the acquisition of ischemic tolerance, thereby identifying this specific kinase as a critical component of the innate defensive response of the heart to injury. Importantly, disruption of these cardioprotective phenotypes occurred in the face of preserved activation of PKC $\varepsilon$ in $L c k^{-/-}$mice that were subjected to ischemic preconditioning (Figure $5 \mathrm{~d}$ ) and in PKCe-Lck ${ }^{-/-}$mice (Figure 5, b and c), demonstrating that PKCE activation is unable to produce a cardioprotective effect without its interactions with, and activation of, Lck. These observations provide direct evidence that Lck is not only an obligatory signaling element in ischemic preconditioning and PKCEinduced protection, but also an essential signaling partner of PKCE in the genesis of both of these forms of cardioprotection. The fact that enhanced tolerance to ischemia was congruous with enhanced formation of PKCE-Lck signaling modules, and that disruption of these modules via the ablation of the Lck gene blocked the infarct-sparing effects elicited by two different stimuli, indicates that PKCE-Lck modules serve as a pivotal nexus in cardioprotective signaling and thus may be an important target for therapeutic interventions.

PKCE-Lck module is required for NF- $\kappa B$ activation during cardioprotection. Previous investigations have established activation of NF- $\mathrm{KB}$ as an essential signaling event in the development of preconditioning (36-38). 
These studies have shown that ischemic preconditioning activates NF- $\kappa \mathrm{B}$ via a PKC-dependent mechanism $(37,38)$. Thus, to gain insights into the mechanisms that are regulated by PKCE-Lck modules, we examined the activation of NF- $\mathrm{\kappa B}$. In agreement with our previous results $(37,38)$, we found that ischemic preconditioning elicited a marked activation of NF- $\mathrm{KB}$ in wildtype mice (NF-KB DNA-binding activity: $378.5 \% \pm 8.8 \%$ of non-preconditioned mice; $n=5$ in each group; $P<$ 0.05; Figure 6a, left panel). In $L c k^{-/}$mice, the basal level of NF- $\mathrm{\kappa B}$ activity was lower (data not shown) and the ischemic preconditioning protocol failed to induce activation of NF- $\kappa B$ (Figure 6a, right panel). In parallel to these findings in the ischemic preconditioning experiments, the level of activated NF- $\mathrm{KB}$ was significantly increased in PKCE cardioprotected mice $234.5 \% \pm 5.6 \%$ of wild-type mice; $n=5$ in each group; $P<0.05$; Figure $6 \mathrm{~b}$, left panel). However, in PKC $\varepsilon-L c k^{-/-}$mice, the PKC $\varepsilon$ induced activation of NF- $\mathrm{\kappa B}$ was obliterated by deletion of the Lck gene (Figure 6b, right panel). These data illustrate an example of the manner in which PKC $\varepsilon$-Lck signaling modules function to regulate cellular processes (here, the induction of NF- $\mathrm{KB}$ ) that are crucial to the genesis of cardioprotection.

\section{Discussion}

This study is, to our knowledge, the first to demonstrate that PKCE, a serine/threonine kinase, and Lck, a tyrosine kinase, can establish direct protein-protein interactions and function in concert as a signaling module to elicit both biochemical and physiological responses. In the in vitro setting, the formation of this signaling module promoted signal transduction, as documented by the finding that increased PKCE-Lck interactions and PKCe-induced phosphorylation of Lck enhanced the kinase activity of Lck. In the in vivo setting, formation of the PKCE-Lck signaling module was found to be indispensable for the transduction of cardioprotective signals generated by the activation of PKCE. Disruption of this module via deletion of the Lck gene abrogated two different forms of protection against myocardial infarction, ischemic preconditioning, and PKCE-mediated cardioprotection, implying a central function of the PKCE-Lck module in governing the tolerance of the heart to ischemia/reperfusion injury. Taken together, these findings demonstrate a fundamental physiological role for a serine/threonine kinase-tyrosine kinase signaling module in the manifestation of a biological phenotype in vivo.
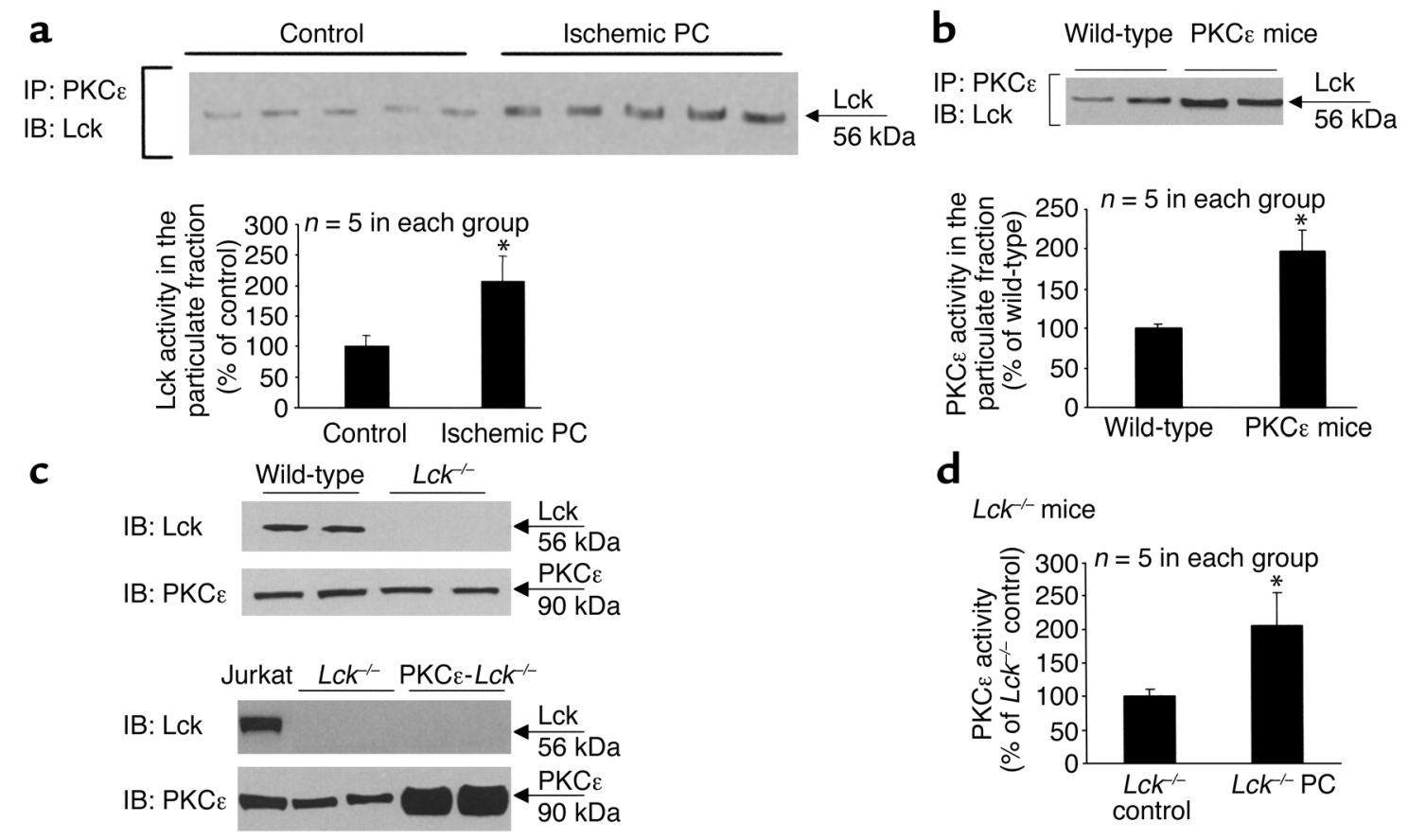

\section{Figure 5}

Ischemic preconditioning (PC) and PKC $\varepsilon$-induced cardioprotection are associated with increased formation of PKCE-Lck signaling modules. (a) Cardiac samples obtained from wild-type mice that underwent an ischemic PC protocol (six cycles of 4-minute coronary occlusion/ reperfusion) exhibited increased colocalization of Lck with PKCE (upper panel) and enhanced Lck phosphorylation activity (lower panel), indicating that ischemic PC was associated with increased formation of PKC $\varepsilon$-Lck signaling modules. ${ }^{*} P<0.05$ vs. control. (b) Myocardial tissue samples obtained from PKCE transgenic mice displayed increased colocalization of Lck with PKCE (upper panel) and enhanced Lck phosphorylation activity (lower panel), indicating that PKCE-induced cardioprotection was concomitant with increased formation of PKC $\varepsilon$-Lck signaling modules. ${ }^{*} P<0.05$ vs. wild-type. (c) Characterization of $L c k^{-/-}$mice and PKCE- $L c k^{-/-}$mice. The PKCE expression in $L c k^{-/-}$mice was similar to that in wild-type mice, indicating that ablation of the $L c k$ gene did not affect PKCE expression (upper panels). In PKC $\varepsilon_{-} L c k^{-/-}$mice, increased expression of PKC $\varepsilon$ was preserved whereas Lck expression was absent (lower panels). (d) Preserved PKC $\varepsilon$ activation by ischemic PC in $L c k^{-/}$mice. Ischemic PC induced significant activation of PKC $\varepsilon$ in $L c k$ mice, indicating that ablation of the $L c k^{-/-}$gene did not affect activation of PKCE. All tissue samples were harvested 30 minutes after ischemic PC. ${ }^{*} P<0.05 \mathrm{vs}$. $L c k^{-/-}$control. Data are mean \pm SEM. 
$\mathbf{a}$

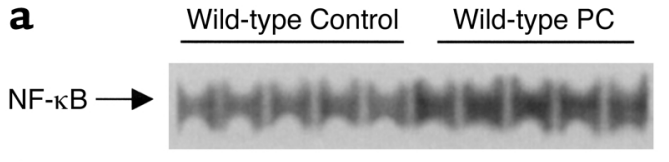

b

Wild-type
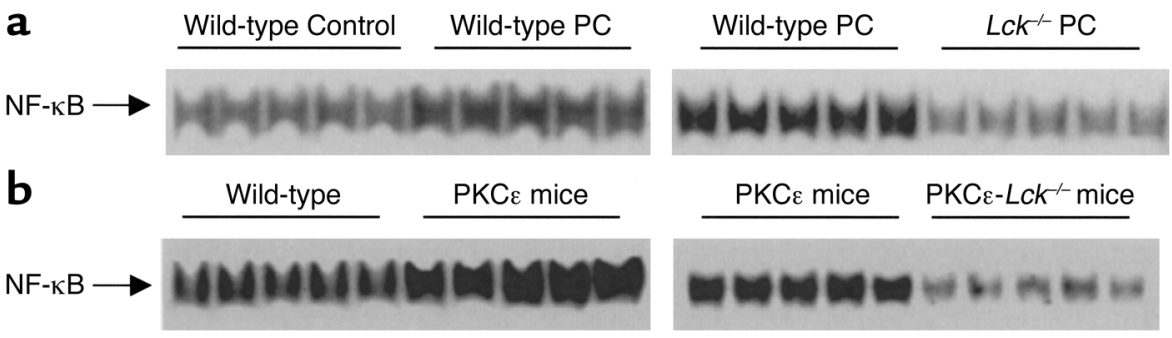

\section{Figure 6}

Ablation of the $L c k$ gene abolishes activation of NF- $K B$ induced by ischemic preconditioning (PC) and by PKCE. The electrophoretic mobility shift assay was used to assess DNA-binding activity of NF- $\mathrm{KB}$ $(37,38)$. (a) Ischemic PC significantly enhanced NF-KB DNA-binding activity, and this activation was completely abolished by ablation of the $L c k$ gene. Nuclear NF-KB DNA-binding activities from five shamoperated and five ischemically preconditioned wild-type mice are shown in the upper left panel, whereas NF-KB DNA-binding activities from five wild-type and five $\mathrm{Lck}^{-/-}$mice that underwent the same ischemic PC protocol are shown in the upper right panel. Super-shift assays confirmed that the complexes contain both the $\mathrm{p} 50$ and $\mathrm{p} 65$ isoforms. Tissue samples were harvested 30 minutes after ischemic PC. (b) Similar to ischemic PC, PKC $\varepsilon$ transgenesis significantly enhanced NF-KB DNA-binding activity, and this activation was completely abolished by ablation of the $L c k$ gene. NF- $\kappa B$ DNA-binding activities from five wild-type and five PKCE mice are shown in the lower left panel, whereas NF-KB DNA-binding activities from five PKC $\varepsilon$ and five PKC $\varepsilon-L c k^{-/-}$mice are shown in the lower right panel.

Both the $\varepsilon$ isoform of the PKC family and the Lck isoform of the Src family have been shown to participate in a variety of cellular processes $(4-8,26,27,29-32)$. However, no evidence is available in any cell type regarding how these two molecules may functionally interact to modulate a biological response. Although the phosphorylation of Lck on serine/threonine and/or tyrosine residues in its $\mathrm{SH} 2, \mathrm{SH} 3$, and carboxyl terminal domains has been suggested as a means to regulate its kinase activity $(26,27)$, a direct molecular modification of Lck by PKC $\varepsilon$ has never been described. By combining MALDI mass spectrometric analysis with site-directed mutagenesis, we identified the serine 166 residue of the Lck SH2 domain as the specific site phosphorylated by PKC $\varepsilon$ in vitro and demonstrated that the physical interactions that occur between the two kinases involve both the catalytic and the regulatory domains of PKCE. While PKCE has been previously shown to interact with other cellular proteins, such as its own receptors (receptors for activated C kinase, RACKs) (5), cytoskeletal proteins (e.g., myristoylated alanine rich $\mathrm{C}$ kinase substrate, MARCKS) (9), and transmembrane channels (e.g., $\mathrm{N}$-type calcium channels) (29), information regarding the direct interactions of PKCE with other signaling kinases is scarce. Specifically, it has not been shown whether PKCE can govern tyrosine phosphorylation-dependent events through direct interaction with a tyrosine kinase. Here, we demonstrate that active PKC $\varepsilon$ is sufficient to phosphorylate Lck, and that this modification is sufficient to induce increased Lck kinase activity and to elicit Lck-mediated regulation of other proteins (e.g., NF-KB). Moreover, we show that this module plays a necessary role in the signaling system that renders the myocardium resistant to ischemic insults.

The concept that kinases are coupled as signaling modules and function collectively to facilitate the manifesta- tion of a physiological phenotype has broad and fundamental biological implications beyond those related to the genesis of cardioprotection. In view of our observations, we propose that the assembly of a functional signaling module containing PKC $\varepsilon$ and Lck underscores what may be a mechanism of signal transduction that is conserved across multiple biological responses in a variety of cell types. The signaling complexes, like the PKC $\varepsilon$ complex characterized by our laboratory (19), which contain within them stimulus-specific modules, like the PKCE-Lck module that is described herein, may serve as a means by which the cell employs multifunctional signaling elements to perform an array of distinct subcellular tasks.

Although the biochemical nature of tissue injury has been extensively documented (1-3), the molecular mechanisms that are responsible for the induction of damage and the development of protective adaptations are not well understood. As a result, there are limited molecular targets for engineering therapeutic measures aimed at preventing ischemic and other forms of cellular injury. A critical first step toward the development of such strategies is to understand the specific mechanisms that underlie endogenous protective processes. The role of PKCE as an essential protective kinase against ischemia was first demonstrated in cardiac tissue $(4,5,11-13)$. It has subsequently been shown that PKC $\varepsilon$ not only mediates protective signals in the heart but also functions as a protective kinase against ischemic injury in the gut (9), as an antiapoptotic kinase in the thyroid (6), and as a key molecule to precondition neurological functions in the brain $(3,29,30)$, supporting a ubiquitous role for this enzyme in alleviating the consequences of ischemia. However, the precise molecular mechanisms by which this kinase exerts its salubrious effects remain largely undefined.

We hereby present evidence to show that cardiac-targeted transgenesis of PKCE, by itself, is sufficient to render the heart resistant to ischemic injury. To our knowledge, this is the first demonstration that isoform-specific activation of a kinase elicits cardioprotection in vivo. Furthermore, our findings demonstrate that PKCE does not function independently in this regard. The interaction of Lck with PKCE, and the consequent phosphorylation and activation of Lck by PKCE, are essential not only for the transduction of the PKCE cardioprotective signal, but also for the subsequent development of protection, as evidenced by the fact that disruption of the PKCE-Lck module by ablation of the Lck gene completely eliminated the 
cardioprotective phenotype conferred by activation of PKCE. Importantly, the obligatory role of the PKCE-Lck signaling module is not limited to PKCE-induced protection, as deletion of the Lck gene also abrogated ischemic preconditioning. Thus, formation of the PKCE-Lck module is crucial for the cardioprotective effects afforded by both chronic (PKCE transgenesis) and acute (ischemic preconditioning) activation of PKC $\varepsilon$. Insofar as ischemic preconditioning represents a highly conserved biological response (having been described in the brain, kidney, gut, skeletal muscle, and liver) $(1-3,9)$ and is the most powerful cardioprotective intervention identified thus far, the present results have broad implications for our understanding of ischemia/reperfusion injury and protection against tissue injury in general.

The identification of PKCE-Lck as an essential module in the genesis of cardioprotection affords novel therapeutic opportunities whereby agents designed to regulate the formation of this module can be used to prevent ischemic injury. This is a departure from previous paradigms that were based on the use of receptor agonists, most of which have indirect and nonselective effects on the PKC signaling system. If, as our data indicate, cardioprotective signaling proceeds via the dynamic interactions of PKCE and Lck, then rationally designed pharmacological or genetic interventions that directly promote the formation of these PKCE-Lck modules would impart specificity and efficacy to therapeutic strategies aimed at protecting the myocardium in patients with ischemic heart disease.

\section{Acknowledgments}

This study was supported in part by the American Heart Association grants EIG-40167N (P. Ping) and AHA-0110053B (T.M. Vondriska); NIH grants HL-63901 (P. Ping), HL-65431 (P. Ping), HL-43151 (R. Bolli), HL-55757 (R. Bolli), HL-68088 (R. Bolli), and NIH 1S10RR11368-01A1 (W.M. Pierce); the Commonwealth of Kentucky Research Challenge Trust Fund; the University of Louisville Research Foundation; and the Jewish Hospital Research Foundation.

1. Bolli, R. 2000. The late phase of preconditioning. Circ. Res. 87:972-983. 2. Williams, R.S., and Benjamin, I.J. 2000. Protective responses in the ischemic myocardium. J. Clin. Invest. 106:813-818.

3. Dawson, V.L., and Dawson, T.M. 2000. Neuronal ischaemic preconditioning. Trends Pharmacol. 21:423-424.

4. Dorn, G.W., II, et al. 1999. Sustained in vivo cardiac protection by a rationally designed peptide that causes $\varepsilon$ protein kinase $C$ translocation. Proc. Natl. Acad. Sci. USA. 96:12798-12803.

5. Gray, M.O., Karliner, J.S., and Mochly-Rosen, D. 1997. A selective $\varepsilon$-protein kinase $\mathrm{C}$ antagonist inhibits protection of cardiac myocytes from hypoxia-induced cell death. J. Biol. Chem. 272:30945-30951.

6. Knauf, J.A., et al. 1999. Involvement of protein kinase C epsilon (PKCE) in thyroid cell death. A truncated chimeric PKCE cloned from a thyroid cancer cell line protects thyroid cells from apoptosis. J. Biol. Chem. 274:23414-23425.

7. Aley, K.O., Messing, R.O., Mochly-Rosen, R., and Levine, J.D. 2000. Chronic hypersensitivity for inflammatory receptor sensitization mediated by the epsilon isozyme of protein kinase C. J. Neurosci. 20:4680-4685.

8. Chow, J.Y., Uribe, J.M., and Barrett, K.E. 2000. A role for protein kinase C epsilon in the inhibitory effect of epidermal growth factor on calciumstimulated chloride secretion in human colonic epithelial cells. J. Biol. Chem. 275:21169-21176.

9. Song, J.C., Hrnjez, B.J., Farokhzad, O.C., and Matthews, J.B. 1999. PKCepsilon regulates basolateral endocytes in human T84 intestinal epithelia: role of F-actin and MARCKS. Am. J. Physiol. Cell Physiol. 277:C1239-C1249.

10. Pass, J.M., et al. 2001. PKC $\varepsilon$ activity induces dichotomous cardiac phenotypes and modulates PKCE-RACK interactions and RACK expression. Am. J. Physiol. Heart Circ. Physiol. 280:H946-H955.

11. Ping, $P$., et al. 1997. Ischemic preconditioning induces selective translocation of PKC isoforms $\varepsilon$ and $\eta$ in the heart of conscious rabbits without subcellular redistribution of total PKC activity. Circ. Res. 81:404-414.

12. Ping, P., et al. 1999. PKC-dependent activation of $\mathrm{p} 44 / \mathrm{p} 42$ MAPKs during myocardial ischemia-reperfusion in conscious rabbits. Am. J. Physiol. Heart Circ. Physiol. 276:H1468-H1481.

13. Liu, G.S., Cohen, M.V., Mochly-Rosen, D., and Downey, J.M. 1999. Protein kinase $\mathrm{C}-\varepsilon$ is responsible for the protection of preconditioning in rabbit cardiomyocytes. J. Mol. Cell. Cardiol. 31:1937-1948.

14. Murry, C.E., Jennings, R.B., and Reimer, K.A. 1986. Preconditioning with ischemia: a delay of lethal cell injury in ischemic myocardium. Circulation. 74:1124-1136.

15. Ytrehus, K., Liu, Y., and Downey, J.M. 1994. Preconditioning protects ischemic rabbit heart by protein kinase $\mathrm{C}$ activation. Am. J. Physiol. Heart Circ. Physiol. 266:H1145-H1152.

16. Dana, A., Skarli, M., Papakrivopoulou, J., and Yellon, D.M. 2000. Adenosine A1 receptor induced delayed preconditioning in rabbits: induction of p38 MAPK activation and Hsp 27 phosphorylation via a tyrosine kinaseand protein kinase C-dependent mechanism. Circ. Res. 86:989-997.

17. Tong, H., Chen, W., Steenbergen, C., and Murphy, E. 2000. Ischemic preconditioning activates phosphatidylinositol-3-kinase upstream of protein kinase C. Circ. Res. 87:309-315.

18. Ray, P.S., et al. 2001. Transgenic overexpression of $\alpha \mathrm{B}$ crystallin confers simultaneous protection against cardiomyocyte apoptosis and necrosis during myocardial ischemia and reperfusion. FASEB J. 15:393-402.

19. Ping, P., Zhang, J., Pierce, W.M., Jr., and Bolli, R. 2001. Functional proteomic analysis of PKC $\varepsilon$ signaling complexes in the normal heart and during cardioprotection. Circ. Res. 88:59-62.

20. Husi, H., Ward, M.A., Choudhary, J.S., Blackstock, W.P., and Grant, S.G.N. 2000. Proteomic analysis of NMDA receptor-adhesion protein signaling complexes. Nat. Neurosci. 3:661-669.

21 . Fraser, I.D., et al. 2000. Assembly of an A-kinase-anchoring protein- $\beta 2$ adrenergic receptor complex facilitates receptor phosphorylation and signaling. Curr. Biol. 10:409-412.

22. McDonald, P.H., et al. 2000. $\beta$-Arrestin 2: a receptor-regulated MAPK scaffold for the activation of JNK3. Science. 290:1574-1577.

23. Vondriska, T.M., Klein, B., and Ping, P. 2001. Use of functional proteomics to investigate $\mathrm{PKC} \varepsilon$-mediated cardioprotection: the signaling module hypothesis. Am. J. Physiol. Heart Circ. Physiol. 280:H1434-H1441.

24. Yasuda, J., Whitmarsh, A.J., Cavanagh, J., Sharma, M., and Davis, R.J. 1999. The JIP group of mitogen-activated protein kinase scaffold proteins. Mol. Cell. Biol. 19:7245-7254.

25. Hunter, T. 2000. Signaling: 2000 and beyond. Cell. 100:113-127.

26. Sicheri, F., and Kuriyan, J. 1997. Structures of Src-family tyrosine kinases. Curr. Opin. Struct. Biol. 7:777-785.

27. Gervais, F.G., Chow, L.M., Lee, J.M., Branton, P.E., and Veilette, A. 1993. The SH2 domain is required for stable phosphorylation of p56 lck at tyrosine 505, the negative regulatory site. Mol. Cell. Biol. 13:7112-7121.

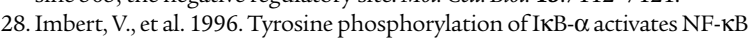
without proteolytic degradation of IK-B- $\alpha$. Cell. 86:787-798.

29. McMahon, T., Anderson, R., Metten, P., Crabbe, J.C., and Messing, R.O. 2000. Protein kinase $C \varepsilon$ mediates up-regulation of $N$-type calcium channels by ethanol. Mol. Pharmacol. 57:53-58.

30. Olive, M.F., and Hodge, C.W. 2000. Co-localization of PKCE with various GABA(A) receptor subunits in the mouse limbic system. Neuroreport. 11:683-687.

31. Jaken, S., and Parker, P.J. 2000. Protein kinase C binding partners. Bioessays. 22:245-254.

32. Ping, P., et al. 1999. Demonstration of selective protein kinase C-dependent activation of Src and Lck tyrosine kinases during ischemic preconditioning in conscious rabbits. Circ. Res. 85:542-550.

33. Sato, T., O'Rourke, B., and Marban, E. 1998. Modulation of mitochondrial ATP-dependent K+ channels by protein kinase C. Circ. Res. 83:110-114.

34. Subramanian, A., Jones, W.K., Gulick, J., Neumann, J., and Robbins, J. 1991. Tissue specific regulation of the alpha-myosin heavy chain gene promoter in transgenic mice. J. Biol. Chem. 266:24613-24620.

35. Guo, Y., et al. 1999. The late phase of ischemic preconditioning is abrogated by targeted disruption of the inducible NO synthase gene. Proc. Natl. Acad. Sci. USA. 96:11507-11512.

36. Maulik, N., Sato, M., Price, B.D., and Das, D.K. 1998. An essential role of $\mathrm{NF}-\mathrm{\kappa B}$ in tyrosine kinase signaling of p38 MAP kinase regulation of myocardial adaptation to ischemia. FEBS Lett. 429:365-369.

37. Xuan, Y.T., et al. 1999. Nuclear factor $\kappa B$ plays an essential role in the late phase of ischemic preconditioning in conscious rabbits. Circ. Res. 84:1095-1099.

38. Li, R.C., et al. 2000. PKC $\varepsilon$ modulates NF- $\mathrm{KB}$ and AP-1 via mitogen-activated protein kinases in adult rabbit cardiomyocytes. Am. J. Physiol. Heart Circ. Physiol. 274:H1679-H1689. 